\title{
Canonical orthonormal Wigner supermultiplet basis
}

\author{
K T Hecht $\dagger+$, R Le Blanct\$ and D J Rowe $\|$ \\ † Physics Department, University of Michigan, Ann Arbor, MI 48109, USA \\ II Physics Department, University of Toronto, Toronto, Ontario, Canada M5S IA?
}

Received 9 April 1986

\begin{abstract}
The explicit construction of an orthonormal basis for states of good spin, isospin and SU(4) Wigner supermultiplet symmetry is given in a Bargmann representation space. A complete set of quantum labels is provided by a $S p(3, M)=U(3)$ complementary symmetry.
\end{abstract}

\section{Introduction}

The classic supermultiplet of Wigner (1937) is the prototype of most of the higher symmetry groups used in modern particle and nuclear physics and continues to play an important role in nuclear spectroscopy. Despite this fact, there has been no complete solution for the Wigner-Racah calculus of this important symmetry. This is related to the internal labelling problem which arises from the insufficiency of the spin and isospin quantum numbers $S$ and $T$ to unambiguously identify all states in the SU(4) D $[S U(2) \times S U(2)]$ reduction and consequently makes it difficult to construct a group theoretically sound orthonormal basis.

In principle, many solutions have been given to the missing labels problem of the Wigner supermultiplet. The integrity basis $\mathrm{SU}(2) \times \mathrm{SU}(2)$ scalars belonging to the enveloping algebra of SU(4) has been analysed by Quesne $(1976,1977)$ who constructed a complete set of commuting operators with the use of the cubic and quartic operators, $\Omega$ and $\Phi$, first introduced by Moshinsky and Nagel (1963), and gave the eigenvalues of these operators for many of the SU(4) irreducible representations of interest in nuclear spectroscopy.

Non-orthogonal bases which lead to simpler additional labels have also been proposed. Draayer (1970) introduced the labels $K_{S}$ and $K_{T}$ and $S, T$-projection techniques in analogy with Elliot's physically meaningful solution of the missing label problem in the $\mathrm{SU}(3) \supset \mathrm{SO}(3)$ scheme. Brunet and Resnikoff (1970) labelled the $\mathrm{SU}(4) \supset[\mathrm{SU}(2) \times \mathrm{SU}(2)]$ basis states through the exponents of the elementary permissible diagrams (Moshinsky and Devi 1969) used in a construction procedure which is of particular interest because it is based on the Littlewood (1950) reduction scheme for the $\mathrm{U}(N) \supset \mathrm{O}(N)$ chain. However, all these solutions are somewhat cumbersome and do not lead to a simple explicit construction of an unambiguous orthonormal $\mathrm{SU}(4) \supset[\mathrm{SU}(2) \times \mathrm{SU}(2)]$ basis.

Recent progress has been made by Deenen and Quesne (1983) and Quesne (1984a, b) who proposed an unambiguous group theoretic solution for the labelling problem of

$¥$ Supported in part by the US National Science Foundation.

$\S$ NSERC Post-Doctoral Fellow.

Supported in part by an NSERC operating grant. 
the general $\mathrm{SU}(N)>\mathrm{SO}(N)$ chain which reflects the operation of Littlewood's branching rule in a much simpler way than the method of permissible diagrams. In the new method, a $\mathrm{U}(N) \supset \mathrm{O}(N)$ basis is constructed for $D$-rowed representations of $\mathrm{U}(N)$ by a classification of states in the fundamental unirreps of $\operatorname{Sp}\left(N D, \Re^{2}\right)$. Using the complementarity of the subgroups $\operatorname{Sp}(D, \Re)$ and $\mathrm{O}(N)$, an equivalence is established between the state labelling problems for $\operatorname{SU}(N) \supset \mathrm{O}(N)$ and $\operatorname{Sp}(D, \Re) \supset \mathrm{U}(D)$ which can then be exploited for the general $D \leqslant(N-1)$-rowed representations of $\operatorname{SU}(N)$. This idea was exploited by Le Blanc and Rowe (1985a,b) to give a very explicit construction of a canonical orthonormal basis for the $\mathrm{SU}(3) \supset \mathrm{SO}(3)$ chain of relevance for the nuclear collective model. The construction uses recent advances (Rowe et al 1984, Rowe 1984, Castaños et al 1984) in the representation theory of the non-compact symplectic groups $\operatorname{Sp}(D, \mathfrak{K})$ whereby an orthonormal basis for $\operatorname{Sp}(D, \mathfrak{R}) \supset \mathrm{U}(D)$ can be given by a very explicit method of construction through the coupling of $U(D)$ intrinsic and collective states using the Biedenharn-Louck (see, e.g., Louck 1970, Le Blanc and Rowe 1986) canonical reduction for the $U(D)$ outer products.

It is the purpose of the present investigation to show that this method can be taken over directly to give a very explicit construction of an unambiguous orthonormal $\mathrm{SU}(4) \supset \mathrm{O}(4) \supset[\mathrm{SU}(2) \times \mathrm{SU}(2)]$ basis for the Wigner supermultiplet scheme. The construction process is a reversal of the Littlewood $S U(4) \downarrow O(4)$ reduction process. The Littlewood branching rule involves the removal of all possible symmetrically zerocoupled pairs from the three-rowed representations of SU(4) to leave $O(4)$ states with definite $S$ and $T$ entirely free of zero-coupled pairs. The zero-coupled pair in this case is a two-particle state coupled to $S=0, T=0$. In the state construction process on the other hand, an $\mathrm{O}(4)$ solid harmonic of definite $S$ and $T$ (the 'intrinsic' state) is coupled with a symmetrically coupled state of $S=0, T=0$ pairs (the 'collective' state) to make a state of good SU(4) symmetry. The equivalence between the $S U(4) \supset O(4)$ and $\mathrm{Sp}(3, \mathfrak{H}) \supset \mathrm{U}(3)$ chains is then exploited through the analogous symplectic state construction (Rosensteel and Rowe 1980) whereby an $\operatorname{Sp}(3, \mathfrak{R})$ intrinsic state is coupled with a symmetrically coupled state of collective excitations to make a state of good SU(3) symmetry. A central feature of the $S p(3, \mathfrak{R}) \supset U(3)$ state construction is the $\kappa^{2}$ or overlap matrix which is vital for the construction of an orthonormal basis. Elegant techniques have recently been developed (Rowe et al 1984, Rowe 1984, Castaños et al $1985 \mathrm{a}, \mathrm{b}$ ) for the evaluation of this matrix which therefore also make it possible to give a very explicit construction of an orthonormal basis for the complementary $\mathrm{SU}(4) \supset \mathrm{O}(4)$ chain.

A number of detailed applications and examples are given which show that the powerful recent advances in the construction of the discrete series unirreps of $\operatorname{Sp}(3, \mathbb{H}) \supset$ $\mathrm{U}(3)$ can also lead to a complete implementation of the Wigner-Racah calculus of the $\mathrm{SU}(4) \supset \mathrm{O}(4) \supset[\mathrm{SU}(2) \times \mathrm{SU}(2)]$ symmetry.

\section{An example}

To understand the nature of the labelling problem, it may be instructive to start with a specific example. For this purpose, we consider the supermultiplet representation $\{422\}$. (Note that this is an important representation both in the nuclear $A=12$ and $A=24$ systems.) The conventional way of carrying out the $S U(4) \downarrow O(4) \downarrow S U(2) \times$ $\mathrm{SU}(2)$ reduction to determine the number of occurrences of a particular $(S T)$ pair within a given supermultiplet proceeds via Littlewood's rules. The reduction is given 
for the $\{422\}$ representation in equation (2.2). The rule states that the possible $O(4)$ symmetries $[\lambda]$ contained in a given $\mathrm{SU}(4)\{h\}$ symmetry are given by the tableaux which remain after the removal from the original tableau of all the possible symmetrically coupled zero-coupled pairs. The zero-coupled pair in this case is a two-particle state coupled to $S=0$ and $T=0$ belonging to an $S U(4)$ representation $\{2\}$. The symmetrically coupled states of $n$ such pairs belong to SU(4) representations labelled by tableaux $\left\{n_{1} n_{2} n_{3}\right\}$ with only even values for $n_{1}, n_{2}$ and $n_{3}$ and $n_{1}+n_{2}+n_{3}=2 n$.

However, in this reduction process non-standard $O(4)$ tableaux appear with as many as three rows. They must therefore be converted to standard tableaux with at most two rows through the use of modification rules. For $\mathrm{O}(4)$ the modification rules are simply

(i)

$$
\begin{aligned}
& {\left[\lambda_{1} \lambda_{2} 2\right] \equiv-\left[\lambda_{1} \lambda_{2}\right]} \\
& {\left[\lambda_{1} 11\right] \equiv\left[\lambda_{1}\right]} \\
& {\left[\lambda_{1} \lambda_{2} \lambda_{3}\right] \equiv 0 \text { for all other cases with } \lambda_{3} \neq 0}
\end{aligned}
$$

Thus for the $\{422\}$ representation we obtain

(removal of)

$$
\begin{aligned}
\{422\} \downarrow & {[422](\equiv-[422]) } \\
& +[42]+[321](\equiv 0)+[222](\equiv-[22]) \\
& +[22] \\
& +[22]+[31]+[4] \\
& +[2] \\
& +[2] \\
& +[0]
\end{aligned}
$$

Note that the application of rule (i) eliminates two allowed two-rowed tableaux in our example, while rule (iii) eliminates the tableau $\{321\}$.

The final $\mathrm{O}(4) \downarrow \mathrm{SU}(2) \times \mathrm{SU}(2)$ reduction is given through the simple rule

$$
\begin{gathered}
\mathrm{O}(4) \downarrow \mathrm{SU}(2) \times \mathrm{SU}(2):\left[\lambda_{1} \lambda_{2}\right] \downarrow(S T)=\left(\frac{1}{2}\left(\lambda_{1}+\lambda_{2}\right), \frac{1}{2}\left(\lambda_{1}-\lambda_{2}\right)\right)+\left(\frac{1}{2}\left(\lambda_{1}-\lambda_{2}\right), \frac{1}{2}\left(\lambda_{1}+\lambda_{2}\right)\right) \\
:\left[\lambda_{1} 0\right] \downarrow(S T)=\left(\frac{1}{2} \lambda_{1}, \frac{1}{2} \lambda_{1}\right) \quad \text { for } \lambda_{2}=0 .
\end{gathered}
$$

The $\{422\}$ multiplet thus contains the $(S T)$ values $(22),(21),(12),(20),(02)$ and $(00)$ once each whereas $(S T)=(11)$ has a double occurrence.

The construction of states in the $S U(4) \supset O(4)$ basis is the reverse of the above Littlewood reduction: first, a $\left(\lambda_{1}+\lambda_{2}\right)$-particle state of $S U(4)$ and $O(4)$ symmetries $\left\{\lambda_{1} \lambda_{2} 0\right\}$ and $\left[\lambda_{1} \lambda_{2}\right]$ respectively is constructed which is entirely free of zero-coupled $((S T)=(00))$ pairs, i.e. it is a generalised harmonic polynomial (solid harmonic) $\mathcal{Y}_{\left[\lambda_{1} \lambda_{2} \lambda_{2} \lambda_{(1}\right]} \equiv \mathcal{Y}_{(S T)}^{\left\{\lambda_{1} \lambda_{2}\right)^{0\}}}$ in the four-dimensional space (cf $\S 3$ ). This generalised solid harmonic is then combined with a $2 n$-particle state $Z_{(00)}^{\{n\}}(A)$ of symmetry $\left\{n_{1} n_{2} n_{3}\right\}$ built from $n$ symmetrically coupled $(S T)=(00)$ pair states $A_{(00)}^{\{2\}}$ :

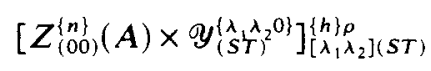

where, in (2.4), the square bracket denotes the $\mathrm{SU}(4) \supset \mathrm{O}(4) \supset[\mathrm{SU}(2) \times \mathrm{SU}(2)]$ coupling

$$
\begin{array}{llllll}
\mathrm{SU}(4): & \{n\} & \times & \{\lambda\} & \rightarrow & \{h\} \\
\mathrm{O}(4): & {[0]} & \times & {[\lambda]} & \rightarrow & {[\lambda]} \\
\mathrm{SU}(2) \times \mathrm{SU}(2): & (00) \times & (S T) \rightarrow & (S T)
\end{array}
$$


where the $\mathrm{O}(4) \supset[\mathrm{SU}(2) \times \mathrm{SU}(2)]$ coupling is actually trivial. Note that a three-rowed tableau $\{h\}$ can in general occur with a multiplicity $g$ in the product $\{n\} \times\{\lambda\}$ necessitating the multiplicity label $\rho(\rho=1,2, \ldots, g)$. (We will assume here that the label $\rho$ is in correspondence with Biedenharn and Louck's canonical resolution of the outermultiplicity problem in terms of operator patterns (Louck 1970, Le Blanc and Rowe 1986).)

The transformation of the non-orthonormal basis (2.4) to an orthonormal basis of states is greatly facilitated by considering the image of the above states in a Bargmann space and by using the Chacón-Moshinsky-Quesne complementarity theorem (Moshinsky and Quesne 1970,1971). The theorem states that, in a Bargmann space of $N \times D$ complex variables $z_{D N}$ or equivalently in the space of the simple $N D$-dimensional harmonic oscillator, states that belong to a unirrep $\langle N / 2(\lambda)\rangle$ of $\operatorname{Sp}(D, \Re)$ also belong to a unirrep $[\lambda]$ of $O(N)$, where we define

$$
N / 2(\lambda) \equiv\left(\lambda_{1}+N / 2, \lambda_{2}+N / 2, \ldots, \lambda_{d}+N / 2\right) .
$$

Likewise, states that belong to a unirrep $\{N / 2(h)\}$ of $\mathrm{U}(D)$ also belong to a unirrep $\{h\}$ of $\mathrm{U}(N)$ (Biedenharn et al 1967).

As a consequence of the complementarity theorem, $\mathrm{U}(D) \times \mathrm{O}(N)$ states for the chain

$$
\left[\begin{array}{ccc}
\mathrm{Sp}(D, \mathscr{H}) & \supset & \mathrm{U}(D) \\
\langle N / 2(\lambda)\rangle & \{n\}, \rho & \{N / 2(h)\}
\end{array}\right] \times \begin{gathered}
\mathrm{O}(N) \\
{[\lambda]}
\end{gathered}
$$

can be identified with

$$
\left[\begin{array}{ccc}
\mathrm{U}(N) & \supset & \mathrm{O}(N) \\
\{h\} & \{n\}, \rho & {[\lambda]}
\end{array}\right] \times \begin{gathered}
\mathrm{U}(D) \\
\{N / 2(h)\}
\end{gathered}
$$

basis states. The state labelling problem for the chain $\mathrm{U}(N) \supset \mathrm{O}(N)$ is thus identified with the $\operatorname{Sp}(D, \Re) \supset \mathrm{U}(D)$ labelling problem which has been fully studied by Rowe $e t$ al (1985). For the supermultiplet problem, we have $N=4$ and $D=3$ for the most general unirrep of $\mathrm{SU}(4) \supset \mathrm{O}(4)$. Details of the construction in the Bargmann space will be given in the following section. Thus in the Bargmann space, the $\mathrm{SU}(4) \supset \mathrm{O}(4) \supset$ $[\mathrm{SU}(2) \times \mathrm{SU}(2)]$ coupling in $(2.4)$ will become a complementary $\mathrm{U}(3) \times \mathrm{O}(4) \supset$ $[\mathrm{SU}(2) \times \mathrm{SU}(2)]$ coupling and the orthonormal Bargmann states (see equations (3.15), (3.22), (3.23) and (3.25)) will be denoted

$$
\left\langle z \mid \begin{array}{c}
\{h\}\{n\} \rho m \\
{[\lambda] S M_{S} T M_{T}}
\end{array}\right\rangle
$$

where $m$ stands for a U(3) labelling scheme such as a Gel'fand pattern. The expression lw or hw in the place of $m$ in (2.8) will signify that the state is a U(3) lowest or highest weight respectively.

(The notation introduced in (2.8) will be the standard notation in the following, i.e. all upper tensorial labels refer to the transformation properties of the states under $\mathrm{U}(3)$ (thus $U(4)$ ) while the lower ones refer to their $\mathrm{O}(4)$ (thus $\operatorname{Sp}(3, \mathfrak{H})$ ) properties. Similarly, a square bracket

$$
\left[\Psi_{1} \times \Psi_{2}\right]_{(S T)}^{\{h\}}
$$

will denote the $U(3) \times[S U(2) \times S U(2)]$ coupling of the enclosed tensors $\Psi$,

$$
\left[\Psi_{1} \times \Psi_{2}\right]_{(s T)}
$$


will denote their $[\mathrm{SU}(2) \times \mathrm{SU}(2)]$ coupling only while

$$
\left[\Psi_{1} \times \Psi_{2}\right]^{\{h\}}
$$

will denote their $\mathrm{U}(3)$ coupling only.)

The states (2.4) are generally non-orthonormal. Also, since $\{n\}$ and $\rho$ cannot be associated directly with the eigenvalues of Hermitian operators, they merely serve as labels (but see the discussion following equation (3.23)). At this stage, however, it appears from the branching rule that the construction procedure of equation (2.4) might be invalid. In the $\{422\}$ multiplet there are two independent $(S T)=(11)$ states corresponding to the two possible $\{n\}$ values $\{222\}$ and $\{42\}$. However, only a single occurrence is predicted for the state $(S T)=(20)$ (or $(02)$ ), and yet there seem to be two ways of constructing such a state through polynomials $Z^{\{n\}}$ with $\{n\}=\{4\}$ or $\{22\}$. Is there an inconsistency?

The answer comes at once from evaluation of the overlap matrix $\kappa^{2}$. For given $[\lambda]$ and $\{h\}$, the overlap matrix $\kappa^{2}([\lambda] ;\{h\})$ for the non-orthonormal basis (2.4) will be defined by (see equations (3.15) and (3.19))

$$
\kappa_{\{n\} \rho,\left\{n^{\prime}\right\} \rho^{\prime}}^{2}([\lambda] ;\{h\})=\left(\begin{array}{c|c}
\{h\}\{n\} \rho m & \{h\}\left\{n^{\prime}\right\} \rho^{\prime} m \\
{[\lambda] S M_{S} T M_{T}} & {[\lambda] S M_{S} T M_{T}}
\end{array}\right) .
$$

This matrix is evaluated very quickly using the techniques developed for the complementary $\operatorname{Sp}(3, \Re) \supset \mathrm{U}(3)$ chain (Rowe et al 1984, Rowe 1984). We find for the states of $\mathrm{O}(4)$ symmetry [22] $((S T)=(20)$ or $(02))$ of our example that the $\kappa^{2}$ matrix has the numerical value (for an ordered $\{n\}$ basis $\{4\},\{22\}$ )

$$
\kappa^{2}([\lambda]=[22] ;\{h\}=\{422\})=\left(\begin{array}{cc}
4 & -2 \sqrt{ } 10 \\
-2 \sqrt{ } 10 & 10
\end{array}\right)
$$

which has diagonal form

$$
\kappa_{\text {diag }}^{2}=\left(\begin{array}{rr}
14 & 0 \\
0 & 0
\end{array}\right)
$$

One of the eigenvalues of $\kappa^{2}$ is zero. Thus, one of the two states has zero norm and there exists only one independent state with $(S T)=(20)$ (or $(02)$ ). The $\kappa^{2}$ matrix immediately signals this important fact.

The $\kappa^{2}$ matrix can also be used to understand the disappearance of the subgroup $O(4)$ unirrep [42] in our example. In fact, the $\kappa^{2}$ matrix shows in a very general way that the $O(4)$ symmetry $\left[\lambda_{1} \lambda_{2}\right]$ does not exist in the Wigner supermultiplet symmetry $\left\{\lambda_{1} \lambda_{2} 2\right\}$. For one-dimensional matrices $\kappa^{2}$, the value $\kappa_{\{n\}\{n\}}^{2}$ is obtained from the eigenvalues $\Omega$ of the $U(3)$ scalar operator of Rowe (1984) through the recursive relation

$$
\begin{aligned}
&\left.\frac{\kappa_{\{n\}\left\{n^{\prime}\right\}}^{2}\left(\left[\lambda_{1} \lambda_{2}\right]\right.}{\kappa_{\{n\}\{n\}}^{2}\left(\left[\lambda_{1} \lambda_{2}\right] ;\right.} ;\{h\}\right) \\
&=\left(\Omega\left(\lambda n^{\prime} h^{\prime}\right)-\Omega(\lambda n h)\right) \\
&=\frac{1}{2}\left(\Delta h_{1}^{2}+\Delta h_{2}^{2}+\Delta h_{3}^{2}\right)+\left(h_{1}-h_{3}-1\right) \Delta h_{1}+\left(h_{2}-h_{3}-2\right) \Delta h_{2} \\
&-3 \Delta h_{3}+2 h_{3}+4+\xi(n)
\end{aligned}
$$

with

$$
\begin{array}{ll}
\xi(n)=-n_{1} & \Delta n_{1}=2 \\
\xi(n)=-n_{2}+1 & \Delta n_{2}=2 \\
\xi(n)=-n_{3}+2 & \Delta n_{3}=2
\end{array}
$$


where $\Delta h_{i}=h_{i}^{\prime}-h_{i}, \Delta n_{i}=n_{i}^{\prime}-n_{i}$ and the number four gives the dimension of the spin-isospin space. With $\{n\}=\{0\}$, we see at once that

$$
\begin{aligned}
\frac{\kappa_{\{2\}\{2\}}^{2}\left(\left[\lambda_{1} \lambda_{2}\right] ;\left\{\lambda_{1} \lambda_{2} 2\right\}\right)}{\kappa_{\{0\}\{0\}}^{2}\left(\left[\lambda_{1} \lambda_{2}\right] ;\left\{\lambda_{1} \lambda_{2}\right\}\right)} & =\left[\frac{1}{2}\left(0+0+2^{2}\right)+0+0-3 \times 2+0+4+0\right] \\
& =0
\end{aligned}
$$

regardless of the values of $\lambda_{1}, \lambda_{2}$. The $O(4)$ state $\left[\lambda_{1} \lambda_{2}\right]$ in the supermultiplet $\left\{\lambda_{1} \lambda_{2} 2\right\}$ has a zero norm in accord with the branching rule obtained from the Littlewood reduction (cf equation (2.1)).

\section{State construction in a $3 \times 4$-dimensional Bargmann space}

The simplest way to give the explicit construction of the states (2.3) is through states of a $3 \times 4$-dimensional oscillator basis in a Bargmann space of $3 \times 4$ complex variables

$$
z_{\alpha j} \quad \alpha=1,2,3 ; j=1,2,3,4
$$

where $z_{\alpha j}$ serve as oscillator creation operators while the $\partial / \partial z_{\alpha j}$ are the corresponding annihilation operators with respect to the Bargmann measure. Note that $\alpha$ ranges from 1-3 to make it possible to construct the states of three-rowed tableau symmetry needed for the most general SU(4) symmetry. The four possible orientations of spin and isospin are given by

$$
\begin{aligned}
& |j=1\rangle=|++\rangle=\left|m_{s}=+\frac{1}{2}, m_{t}=+\frac{1}{2}\right\rangle \\
& |j=2\rangle=|+-\rangle=\left|m_{s}=+\frac{1}{2}, m_{t}=-\frac{1}{2}\right\rangle \\
& |j=3\rangle=|-+\rangle=\left|m_{s}=-\frac{1}{2}, m_{l}=+\frac{1}{2}\right\rangle \\
& |j=4\rangle=|--\rangle=\left|m_{s}=-\frac{1}{2}, m_{t}=-\frac{1}{2}\right\rangle .
\end{aligned}
$$

We could also express these through a cartesian basis

$$
\begin{aligned}
& \left|e_{1}\right\rangle=(1 / \sqrt{ } 2)(|++\rangle+|--\rangle) \\
& \left|e_{2}\right\rangle=(\mathrm{i} / \sqrt{ } 2)(|++\rangle-|--\rangle) \\
& \left|e_{3}\right\rangle=(\mathrm{i} / \sqrt{ } 2)(|+-\rangle+|-+\rangle) \\
& \left|e_{4}\right\rangle=(1 / \sqrt{ } 2)(|+-\rangle-|-+\rangle) .
\end{aligned}
$$

The zero-coupled pair states are then

$$
\begin{aligned}
z_{\alpha} z_{\beta} & =\sum_{i} z_{\alpha e_{t}} z_{\beta e_{i}}=A_{\alpha \beta}(z) \\
& =\left(z_{\alpha++} z_{\beta--}+z_{\alpha--} z_{\beta++}-z_{\alpha+-} z_{\beta-+}-z_{\alpha-+} z_{\beta+-}\right) \\
& =2 \sum_{m_{1} m_{l}}\left\langle\frac{1}{2} m_{s} \frac{1}{2}-m_{s} \mid 00\right\rangle\left\langle\frac{1}{2} m_{t} \frac{1}{2}-m_{t} \mid 00\right\rangle z_{\alpha m_{t} m_{t}} z_{\beta-m_{\iota}-m_{t}}
\end{aligned}
$$

where the last form shows the $S=0, T=0$ character of the pair. We will take advantage of the isomorphism $\mathrm{SO}(4) \sim \mathrm{SU}(2) \times \mathrm{SU}(2)$ and therefore of the angular momentum calculus by consistently using the spin-isospin basis (3.2) and the corresponding $S T$ coupling instead of the cartesian basis (3.3) and a corresponding $O(4)$ coupling (but see the discussion following equation (4.7)). 
The first step in the $\mathrm{SU}(4) \supset \mathrm{SU}(2) \times \mathrm{SU}(2)$ state construction process involves the construction of the states entirely free of $(S T)=(00)$ pair states, i.e. generalised solid harmonics $Y_{S S T}^{\{\lambda\}},(z)$ in the four-dimensional Bargmann space. These must satisfy the equation (Lohe and Hurst 1971)

$$
\nabla_{\alpha \beta} y_{\{S T\}}^{\{\lambda\}}(z)=\frac{\partial^{2}}{\partial z_{\alpha} \partial z_{\beta}} y_{(S T)}^{\{\lambda\}}(z)=0
$$

\subsection{One-rowed solid harmonics $\left[\lambda_{1}\right]$}

The simplest are the totally symmetric solid harmonics of symmetry $\left[\lambda_{1}\right]$ which can be constructed in terms of a single four-dimensional variable $z_{1}$ only when highest weight in the complementary $\mathrm{U}(3)$ group action. The condition (3.5) leads to the normalised solid harmonic (with $S=T=\frac{1}{2} \lambda_{1}$ )

$$
\begin{aligned}
\mathscr{Y}_{S M_{S} T M_{T}}^{\left\{\lambda_{1}\right\} \mathrm{hW}}(z)= & \left(\frac{\left(\frac{1}{2} \lambda_{1}+M_{S}\right) !\left(\frac{1}{2} \lambda_{1}+M_{T}\right) !\left(\frac{1}{2} \lambda_{1}-M_{S}\right) !\left(\frac{1}{2} \lambda_{1}-M_{T}\right) !}{\lambda_{1} !}\right)^{1 / 2} \\
& \times \sum_{a=M_{S}+M_{T}}^{\min \left(S+M_{S} T+M_{T}\right)} \frac{\left(z_{1++}\right)^{a}}{a !} \frac{\left(z_{1+-}\right)^{\lambda_{1} / 2+M_{2}-a}}{\left(\frac{1}{2} \lambda_{1}+M_{S}-a\right) !} \frac{\left(z_{1-+}\right)^{\lambda_{1} / 2+M_{T}-a}}{\left(\frac{1}{2} \lambda_{1}+M_{T}-a\right) !} \frac{\left(z_{1--}\right)^{a-M_{S}-M_{T}}}{\left(a-M_{S}-M_{T}\right) !}
\end{aligned}
$$

simplifying to

$$
\mathscr{Y}_{S S T T}^{\left\{\lambda_{1}\right\} \mathrm{hw}}(z)=\frac{z_{++}^{\lambda_{1}}}{\sqrt{\lambda_{1}} !}
$$

when $M_{S}=S$ and $M_{T}=T$.

\subsection{Two-rowed solid harmonics $\left[\lambda_{1} \lambda_{2}\right]$}

For $S>T$, we define

$$
\begin{aligned}
& \mathcal{Y}_{\left(S=\left\{\lambda_{1}+\lambda_{2}\right) / 2, T=\left(\lambda_{1}-\lambda_{2}\right) / 2\right.}^{\left(\lambda_{1}\right)}(z) \\
& =\frac{N\left(\lambda_{1}-\lambda_{2}, \lambda_{2}, 0\right)}{N\left(\lambda_{1}-\lambda_{2}, 0,0\right) N\left(0, \lambda_{2}, 0\right)}\left[\mathscr{Y}_{\left(S_{1}=T^{2}=\left(\lambda_{1}-\lambda_{2}\right) / 2\right)}^{\left\{\lambda_{1}-\lambda_{2}, 0.0\right\}}(z) \times \mathcal{Y}_{\left(S_{2}=\lambda_{2}, T=0\right)}^{\left\{\lambda_{2}, \lambda_{2}, 0\right\}}(z)\right]_{(s T)}
\end{aligned}
$$

where

$$
\mathcal{Y}_{S=M_{S}=\lambda_{2}, T=M_{T}=0}^{\left\{\lambda_{2}, \lambda_{2}, 0\right\} h w}(z)=N\left(0, \lambda_{2}, 0\right)\left|\begin{array}{ll}
z_{1++} & z_{1+-} \\
z_{2++} & z_{2+-}
\end{array}\right|^{\lambda_{2}}
$$

and where $N(a, b, c)$ is the normalisation factor for a $\mathrm{U}(3) \times \mathrm{SU}(4)$ highest weight Bargmann polynomial of dual rank $\{a+b+c, b+c, c\}$, i.e. the Bargmann norm of

$$
N(a, b, c) z_{11}^{a}\left|\begin{array}{ll}
z_{11} & z_{12} \\
z_{21} & z_{22}
\end{array}\right|^{b}\left|\begin{array}{lll}
z_{11} & z_{12} & z_{13} \\
z_{21} & z_{22} & z_{23} \\
z_{31} & z_{32} & z_{33}
\end{array}\right|
$$

is unity when (Moshinsky 1962)

$$
N(a, b, c)=\left(\frac{(a+1)(b+1)(a+b+2)}{(a+b+c+2) !(b+c+1) ! c !}\right)^{1 / 2} .
$$


When $M_{S}=S$ and $M_{T}=T,(3.7 a)$ simplifies to

$$
y_{S S T T}^{\left(\lambda_{1} \lambda_{2} 0\right\} \mathrm{hw}}(z)=N\left(\lambda_{1}-\lambda_{2}, \lambda_{2}, 0\right) z_{1++}^{\lambda_{1}-\lambda_{2}}\left|\begin{array}{cc}
z_{1++} & z_{1+-} \\
z_{2++} & z_{2+-}
\end{array}\right|^{\lambda_{2}} .
$$

Similarly, for $S<T$, we independently define

$\mathcal{Y}_{\left(S=\left(\lambda_{1}-\lambda_{2}\right) / 2, T=\left(\lambda_{1}+\lambda_{2}\right) / 2\right)}^{\left\{\lambda_{1}\right)}(z)$

$$
=\frac{N\left(\lambda_{1}-\lambda_{2}, \lambda_{2}, 0\right)}{N\left(\lambda_{1}-\lambda_{2}, 0,0\right) N\left(0, \lambda_{2}, 0\right)}\left[\mathscr{Y}_{\left(S=T=\left(\lambda_{1}-\lambda_{2}\right) / 2\right)}^{\left(\lambda_{1}-\lambda_{2}, 0,0\right\}}(z) \times \mathscr{Y}_{\left(S=0, T=\lambda_{2}\right)}^{\left\{\lambda_{2}, \lambda_{2}, 0\right\}}(z)\right]_{(S T)}
$$

where

$$
y_{S=M_{S}=0, T=M_{T}=\lambda_{2}}^{\left\{\lambda_{2}, \lambda_{2}, 0\right\} \mathrm{hw}}(z)=N\left(0, \lambda_{2}, 0\right)\left|\begin{array}{ll}
z_{1++} & z_{1-+} \\
z_{2++} & z_{2-+}
\end{array}\right|^{\lambda_{2}} .
$$

The independent definition of the $S<T$ solid harmonic is necessitated by the fact that we do not use lowering (raising) techniques to build a basis for the SU(4) unirrep $\{h\}$ from its highest (lowest) weight state. Here, the basis is constructed $a b$ initio and the matrix elements of the SU(4) generators are calculated afterwards, as in $\S 5$. Since the states $(3.8 a)$ and $(3.8 b)$ correspond to different $S T$ quantum numbers, they have to be defined independently. When $M_{S}=S$ and $M_{T}=T,(3.7 b)$ simplifies to

$$
\mathscr{Y}_{S S T T}^{\left\{\lambda_{1} \lambda_{2} 0\right\} h w}(z)=N\left(\lambda_{1}-\lambda_{2}, \lambda_{2}, 0\right) z_{1++}^{\lambda_{1}-\lambda_{2}}\left|\begin{array}{ll}
z_{1++} & z_{1-+} \\
z_{2++} & z_{2-+}
\end{array}\right|^{\lambda_{2}} \text {. }
$$

The states (3.7) can be given the alternative but equivalent expression $\mathscr{Y}_{\left(S=\left(\lambda_{1} \pm \lambda_{2}\right) / 2(z), T=\left(\lambda_{1} \mp \lambda_{2}\right) / 2\right)}^{\left\{\lambda_{1} \lambda_{2} 0\right\} h w}(z)=\left[\mathscr{Y}_{\left(\lambda_{1} / 2, \lambda_{1} / 2\right)}^{\left\{\lambda_{1}\right\}}\left(z_{1}\right) \times \mathcal{Y}_{\left(\lambda_{2} / 2, \lambda_{2} / 2\right)}^{\left\{\lambda_{2}\right\}}\left(z_{2}\right)\right]_{(S, T)}$

most useful in actual computations.

\subsection{Three-rowed solid harmonics $\left[\lambda_{1}\right]^{*} \equiv\left[\lambda_{1} 11\right]$}

The modification rule (ii) of equation (2.1) indicates that there are solid harmonics of symmetry $\left[\lambda_{1}\right]^{*} \equiv\left[\lambda_{1} 11\right]$ with $S=T=\frac{1}{2} \lambda_{1}$. This is verified by the explicit construction (similar to the construction (Quesne 1984b, Le Blanc and Rowe 1985a, b) of an $\mathrm{SO}(3)$ pseudo-vector of $\mathrm{O}(3)$ character $\left.[L]^{*}=[L 1]\right)$

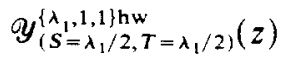

$$
=\frac{N\left(\lambda_{1}-1,0,1\right)}{N\left(\lambda_{1}-1,0,0\right) N(0,0,1)}\left[\mathscr{Y}_{\left(S=T=\left(\lambda_{1}-1\right) / 2\right)}^{\left\{\lambda_{1}-1,0,0\right) \mathrm{hw}}(z) \times \mathcal{Y}_{(S=T=1 / 2)}^{\{1,1,1\} \mathrm{hw}}(z)\right]_{(S T)}
$$

where

$$
\mathcal{Y}_{\left(\boldsymbol{S}=M_{S}=\boldsymbol{T}=M_{T}=1 / 2\right)}^{\{1,1,1\} \mathrm{hW}}(z)=N(0,0,1)\left|\begin{array}{lll}
z_{1++} & z_{1+-} & z_{1-+} \\
z_{2++} & z_{2+-} & z_{2-+} \\
z_{3++} & z_{3+-} & z_{3-+}
\end{array}\right| .
$$

When $M_{S}=S$ and $M_{T}=T,(3.11)$ simplifies to

$$
Y_{S S T T}^{\left[\lambda_{1}, 1,1\right\} \mathrm{hw}}(z)=N\left(\lambda_{1}, 0,1\right) z_{1++}^{\lambda_{1}^{-1}}\left|\begin{array}{lll}
z_{1++} & z_{1+-} & z_{1-+} \\
z_{2++} & z_{2+-} & z_{2-+} \\
z_{3++} & z_{3+-} & z_{3-+}
\end{array}\right| .
$$


The state (3.11) can be given the alternative but equivalent expression

$$
\begin{aligned}
\mathscr{Y}_{\left(S=\lambda_{1} / 2, T=\lambda_{1} / 2\right)}^{\left\{\lambda_{1}, 1,1\right\} \text { hw }} & (z) \\
= & (-1 / \sqrt{ } 2)\left[\mathscr{Y}_{\left(\lambda_{1} / 2, \lambda_{1} / 2\right)}^{\left\{\lambda_{1}\right\}}\left(z_{1}\right) \times\left[\mathscr{Y}_{(1 / 2,1 / 2)}^{\{1\}}\left(z_{2}\right) \times \mathcal{Y}_{(1 / 2,1 / 2)}^{\{1\}}\left(z_{3}\right)\right]_{(10)}\right]_{(S T)} \\
& +(1 / \sqrt{ } 2)\left[\mathcal{Y}_{\left(\lambda_{1} / 2, \lambda_{1} / 2\right)}^{\left\{\lambda_{1}\right\}}\left(z_{1}\right) \times\left[\mathcal{Y}_{(1 / 2,1 / 2)}^{\{1\}}\left(z_{2}\right) \times \mathcal{Y}_{(1 / 2,1 / 2)}^{\{1\}}\left(z_{3}\right)\right]_{(01)}\right]_{(S T)}
\end{aligned}
$$

most useful in actual computations.

As indicated by the modification rules, attempts to make other three-rowed fourdimensional solid harmonics fail. With the exception of the state (3.11), states of three-rowed symmetry always contain some $S T$ zero-coupled pairs.

States (3.6), (3.7), (3.10), (3.11) and (3.13) are all $U(3)$ highest weight states. Lower weight states are easily obtained through standard $U(3)$ lowering techniques (Hecht 1965).

That the above functions $\mathscr{Y}_{(S T)}^{\{\lambda\}}$ are generalised harmonic polynomials in the sense of equation (3.5) is guaranteed by their explicit construction (Louck and Galbraith 1972). They will generally be denoted

$$
\mathcal{Y}_{\{, i, \lambda, \xi\}}^{\{i,\}}
$$

with $\varepsilon=0, S, T=\frac{1}{2}\left(\lambda_{1} \pm \lambda_{2}\right), \frac{1}{2}\left(\lambda_{1} \mp \lambda_{2}\right)$, or with $\varepsilon=1$, (provided $\left.\lambda_{2}=1\right)$ and $S=T=\frac{1}{2} \lambda_{1}$. With these solid harmonics the non-orthonormal states of equation (2.4) can now be constructed:

$$
\left(z \mid \begin{array}{c}
\{h\}\{n\} \rho m \\
{[\lambda] S M_{S} T M_{T}}
\end{array}\right)=\left[Z^{\{n\}}(z) \times Y_{S M_{S} T M_{T}}^{\left\langle\lambda_{1} \lambda_{2}{ }^{E}\right\}}(z)\right]^{\{h\} \rho m} .
$$

Note the round bracket on the left ket to signify its non-orthonormality.

The symmetrically coupled polynomials $Z^{\{n\}}$ of zero-coupled pairs $A$ are well known. The $U(3)$ highest weight is given by

$$
\begin{aligned}
\boldsymbol{Z}^{\left\{n_{1} n_{2} n_{3}\right\} \text { hw }}(\boldsymbol{A}(z)) & =\mathcal{N}(x, y, z)\left(z_{1} z_{2}\right)^{x}\left|\begin{array}{ll}
\left(z_{1} z_{1}\right) & \left(z_{1} z_{2}\right) \\
\left(z_{1} z_{2}\right) & \left(z_{2} z_{2}\right)
\end{array}\right| \cdot\left|\begin{array}{lll}
\left(z_{1} z_{1}\right) & \left(z_{1} z_{2}\right) & \left(z_{1} z_{3}\right) \\
\left(z_{1} z_{2}\right) & \left(z_{2} z_{2}\right) & \left(z_{2} z_{3}\right) \\
\left(z_{1} z_{3}\right) & \left(z_{2} z_{3}\right) & \left(z_{3} z_{3}\right)
\end{array}\right| z
\end{aligned}
$$

where (Quesne 1981)

$$
\mathcal{N}(x, y, z)=\frac{1}{2^{(x+z) / 2}[x ! y !]}\left(\frac{(2 x+1) !(2 y+1) !(x+y) !(x+y+1) !(y+z) !}{z !(2 x+2 y+1) !(2 y+2 z+1) !(x+y+z+1) !}\right)^{1 / 2}
$$

and

$$
n_{1}=2 x+2 y+2 z \quad n_{2}=2 y+2 z \quad n_{3}=2 z .
$$

The zero-coupled pairs $z_{\alpha} z_{\beta}$ belong to the basis of generators of the complementary $\operatorname{sp}(3, \mathfrak{R})$ algebra (Rosensteel and Rowe 1980) spanned by

$$
\begin{aligned}
A_{\alpha \beta} & =z_{\alpha} z_{\beta} & & 6 \text { Cartan raising operators } \\
B_{\alpha \beta} & =\frac{\partial^{2}}{\partial z_{\alpha} \partial z_{\beta}} & & 6 \text { Cartan lowering operators } \\
C_{\alpha \beta} & =\frac{1}{2}\left(z_{\alpha} \frac{\partial}{\partial z_{\beta}}+\frac{\partial}{\partial z_{\beta}} z_{\alpha}\right) & & 9 \mathrm{U}(3) \text { generators. } \\
& =z_{\alpha} \frac{\partial}{\partial z_{\beta}}+2 \delta_{\alpha \beta} & &
\end{aligned}
$$


The $O(4)$ solid harmonics $\mathcal{Y}_{(S T)}^{\left\{\lambda, \lambda_{2} \varepsilon\right\} \mathrm{hw}}(z)$ will also be, through equation $(3.5), \operatorname{sp}(3, \Re)$ symplectic highest weight states $\psi^{\left\{h_{1} h_{2} h_{3}\right\} \text { hw }}$ of $U(3)$ rank $\left\{h_{1} h_{2} h_{3}\right\}=\left\{\lambda_{1}, \lambda_{2}, \varepsilon\right\}$ defined by (Rosensteel and Rowe 1980)

$$
\begin{aligned}
& B_{\alpha \beta} \psi^{\left\{h_{1} h_{2} h_{3}\right\} h w}=0 \\
& C_{\alpha \beta} \psi^{\left\{h_{1} h_{2} h_{3}\right\} \text { hw }}=0 \\
& \alpha<\beta \\
& C_{\alpha \alpha} \psi^{\left\{h_{1} h_{2} h_{3}\right\} \text { hw }}=h_{\alpha} \psi^{\left\{h_{1} h_{2} h_{3}\right\} h w} \quad \text { (with no sum on } \alpha \text { ). }
\end{aligned}
$$

Since the $z_{\alpha} z_{\beta}$ are $\operatorname{sp}(3, \Re)$ lowering operators, the polynomials $Z^{\{n\}}$, when applied to the solid harmonic $\mathcal{Y}^{\left\{\lambda_{1} \lambda_{2} \varepsilon\right\}}$, will generate a complete set of states for the $\operatorname{sp}(3, \mathfrak{R})$ discrete series unirrep $2\langle(\lambda)\rangle$ (Rowe et al 1985). The states (3.15) are thus simultaneously of $\mathrm{SU}(4) \supset \mathrm{O}(4) \supset[\mathrm{SU}(2) \times \mathrm{SU}(2)]$ symmetry and of $\mathrm{Sp}(3, \mathfrak{R}) \supset \mathrm{U}(3)$ symmetry, where $\left\{\lambda_{1} \lambda_{2} \varepsilon\right\}$ characterises both the $O(4)$ and $\operatorname{Sp}(3, \Re)$ unirreps while $\{h\}$ characterises both the SU(4) and U(3) unirreps.

The overlap of the non-orthonormal (round bracketed) states (3.15) is given by the $\kappa^{2}$ matrix (Le Blanc and Rowe 1985a)

$$
\kappa_{\{n\} \rho,\left\{n^{\prime}\right\} \rho^{\prime}}^{2}([\lambda] ;\{h\})=\left(\begin{array}{c|c}
\{h\}\{n\} \rho m & \{h\}\left\{n^{\prime}\right\} \rho^{\prime} m \\
{[\lambda] S M_{S} T M_{T}} & {[\lambda] S M_{S} T M_{T}}
\end{array}\right) .
$$

If a $l \times l \kappa^{2}$ matrix is diagonalised by the unitary matrix $U$

$$
U^{\dagger} \kappa^{2} U=\Lambda=\operatorname{diag}\left(\Lambda_{1}, \Lambda_{2}, \ldots, \Lambda_{l}\right)
$$

then we can make the canonical choice

$$
\kappa=U \Lambda^{1 / 2} U^{+} \quad \kappa^{-1}=U^{+} \Lambda^{-1 / 2} U
$$

and define the orthonormal basis

$$
\left.\left|\begin{array}{c|c}
\{h\}\{n\} \rho m \\
{[\lambda] S M_{S} T M_{T}}
\end{array}\right\rangle=\sum_{\left\{n^{\prime}\right\} \rho^{\prime}} \kappa_{\{n\} \rho,\left\{n^{\prime}\right\} \rho^{\prime}}^{-1} \mid \begin{array}{c}
\{h\}\left\{n^{\prime}\right\} \rho^{\prime} m \\
{[\lambda] S M_{S} T M_{T}}
\end{array}\right) .
$$

As already shown by the example of $\S 2$, the power of this $\kappa^{2}$ method is that it automatically reveals problems of overcompleteness (Rowe et al 1985) through the appearance of zero eigenvalues. In those cases where $\kappa^{2}$ has $p$ zero eigenvalues, one may choose to define a basis canonically, i.e. to retain the meaning of the quantum labels $\{n\} \rho$, by truncating the $l \times l$ singular matrix $\kappa^{2}$ to a $(l-p) \times(l-p)$ non-singular matrix $\left(\kappa^{\text {trunc }}\right)^{2}$ where $p$ rows and the corresponding $p$ columns have been deleted. One then defines

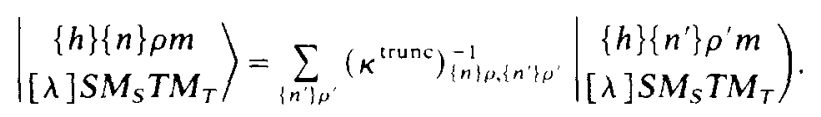

The choice (3.23) has the advantage of explicitly enumerating the subset of the overcomplete non-orthonormal set of states (3.15) used in the construction of the orthornormal states. Note too that the non-orthonormal (round bracketed) state (3.15) with the biggest contribution to the orthonormal state (3.23) is in most cases the one with $\left\{n^{\prime}\right\} \rho^{\prime}=\{n\} \rho$ (Rowe 1984). Thus the quantum labels $\{n\}$ and $\rho$ then still retain a group theoretic significance. Alternatively, to avoid the arbitrariness of choosing which states of the overcomplete set to eliminate, one may restrict to the non-null vectors in the basis in which $\kappa^{2}$ is diagonal and define

$$
\kappa_{i,\{n\} \rho}^{-1}=\Lambda_{i}^{-1 / 2} U_{i\{n\} \rho} \quad \kappa_{\{n\} \rho, 1}=U_{\{n\} \rho, 1}^{*} \Lambda_{i}^{1 / 2} \quad \text { (for } \Lambda_{1} \neq 0 \text { and no sum on } i \text { ). }
$$


The corresponding orthonormal basis (used in this paper) will then be denoted

$$
\left.\left|\begin{array}{c|c|c}
\{h\} \mathrm{im} \\
{[\lambda] S M_{S} T M_{T}}
\end{array}\right\rangle=\sum_{\{n\} \rho} \kappa_{i,\{n\} \rho}^{-1} \mid \begin{array}{c}
\{h\}\{n\} \rho m \\
{[\lambda] S M_{S} T M_{T}}
\end{array}\right) \quad 1 \leqslant i \leqslant l-p .
$$

Finally, it should also be mentioned that the symmetrically coupled raising polynomials $Z^{\{n\}}$ of equation (3.16) are normalised such that

$$
\left[Z^{\{2\}} \times Z^{\{n\}}\right]^{\left\{n^{\prime}\right\}}=\left(\left\{n^{\prime}\right\}\left\|a^{*}\right\|\{n\}\right) Z^{\left\{n^{\prime}\right\}}
$$

where the doubly barred matrix element is the $\mathrm{SU}(3)$ reduced matrix element of the six-dimensional oscillator creation operator of symmetry $\{2\}$ used in Quesne (1981) and Rosensteel and Rowe (1983) and given by

$$
\begin{aligned}
\left(\left\{n^{\prime}\right\}\left\|a^{\dagger}\right\|\{n\}\right) & =\left(\frac{\left(n_{1}+4\right)\left(n_{1}-n_{2}+2\right)\left(n_{1}-n_{3}+3\right)}{2\left(n_{1}-n_{2}+3\right)\left(n_{1}-n_{3}+4\right)}\right)^{1 / 2} \delta_{n_{1}, n_{1}+2} \delta_{n_{2}, n_{2}} \delta_{n_{3}, n_{3}} \\
& +\left(\frac{\left(n_{2}+3\right)\left(n_{1}-n_{2}\right)\left(n_{2}-n_{3}+2\right)}{2\left(n_{1}-n_{2}-1\right)\left(n_{2}-n_{3}+3\right)}\right)^{1 / 2} \delta_{n_{1}^{\prime}, n_{1}} \delta_{n_{2}, n_{2}+2} \delta_{n_{3}, n_{3}} \\
& +\left(\frac{\left(n_{3}+2\right)\left(n_{2}-n_{3}\right)\left(n_{1}-n_{3}+1\right)}{2\left(n_{1}-n_{3}\right)\left(n_{2}-n_{3}-1\right)}\right)^{1 / 2} \delta_{n_{1}, n_{1}, \delta_{n_{2}, n_{2}} \delta_{n_{3}, n_{3}+2}}
\end{aligned}
$$

\section{4. $S U(4) \supset O(4)$ Wigner coefficients}

\section{1. $S U(4) \supset O(4)$ Wigner coefficients for the fundamental tensor}

To show how the very explicit state construction of $\$ 3$ can be used to give a full implementation of the $S U(4) \supset O(4)$ Wigner-Racah calculus, we indicate how the simplest type of SU(4) Wigner coefficient can be calculated in the Bargmann framework. The Bargmann variable $z$ is the fundamental tensor for both complementary $U(3)$ and SU(4) symmetries:

$$
T_{[1] m_{s} / 2, m_{t} / 2}^{\{100\} m}(z)=z_{\alpha m_{s} m_{i}}
$$

where

$$
m=\backslash \begin{array}{lll}
1 & & 0 \\
& 1 &
\end{array} /, \backslash \begin{array}{lll}
1 & & 0 \\
& 0 &
\end{array} /, \backslash \begin{array}{lll}
0 & 0 \\
0
\end{array} / \quad \text { for } \alpha=1,2,3 .
$$

The reduced matrix element (with respect to $U(3) \times[S U(2) \times S U(2)]$ ) in the basis (3.2) can be written as

$$
\begin{aligned}
& \left\langle\begin{array}{c}
\left\{h^{\prime}\right\} j \\
{\left[\lambda^{\prime}\right]\left(S^{\prime} T^{\prime}\right)}
\end{array}\left\|T_{[1](1 / 21 / 2)}^{\{100\}}\right\| \begin{array}{c}
\{h\} i \\
{[\lambda](S T)}
\end{array}\right) \\
& =C\left(\{h\} ;\left\{h^{\prime}\right\}\right)\left\langle\begin{array}{cc||c}
\{h\} i & \{1\} & \left\{h^{\prime}\right\} j \\
{[\lambda](S T)^{\prime}[1](1 / 21 / 2)}
\end{array}\right.
\end{aligned}
$$

where the factor $C$ (independent of the SU(4) subgroup labels) is a normalisation factor usually determined through the orthonormality of the reduced Wigner coefficients. However, for the Bargmann space, $C$ can easily be deduced from normalisation considerations and is given by

$$
C^{2}\left(\{h\} ;\left\{h^{\prime}\right\}\right)=\frac{N^{2}\left(h_{1}-h_{2}, h_{2}-h_{3}, h_{3}\right)}{N^{2}\left(h_{1}^{\prime}-h_{2}^{\prime}, h_{2}^{\prime}-h_{3}^{\prime}, h_{3}^{\prime}\right)} .
$$

(We agree to take the positive square root of the normalisation factor, i.e. $C=+\sqrt{C^{2}}$.) 
To evaluate the reduced matrix element of equation (4.2), it is sufficient to re-express the various terms in the expression

$$
\left[\left[Z^{\{n\}} \times \mathscr{Y}_{(S T)}^{\left\{\lambda_{1} \lambda_{2} \varepsilon\right\}}\right]^{\{h\} \rho} \times T_{(1 / 21 / 2)}^{\{1\}}\right]_{\left(S^{\prime} T^{\prime}\right)}^{\left\{h^{\prime}\right\}}
$$

in the standard form (3.13). The position of $T^{\{1\}}$ in (4.4) is dictated by the standard conventions for reduced matrix elements. A simple recoupling transformation, requiring an SU(3) Racah coefficient (Draayer and Akiyama 1973) and reordering of the $[\mathscr{Y} \times T]$ coupling yields

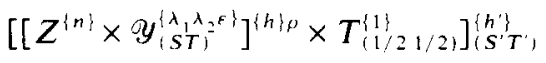

$$
\begin{aligned}
& =\sum_{\left(\lambda_{k} \mu_{k}\right) \rho^{\prime}} U\left(\left(\lambda_{n} \mu_{n}\right)\left(\lambda_{1}-\lambda_{2}, \lambda_{2}-\varepsilon\right)\left(\lambda_{h^{\prime}} \mu_{h^{\prime}}\right)(10) ;\left(\lambda_{h} \mu_{h}\right) \rho_{-}\left(\lambda_{k} \mu_{k}\right)_{-} \rho^{\prime}\right)
\end{aligned}
$$

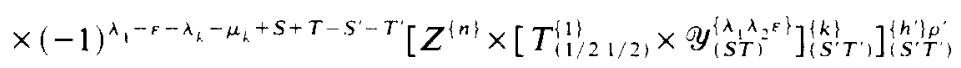

with $\lambda_{h}=h_{1}-h_{2}, \mu_{h}=h_{2}-h_{3}$, etc. Draayer and Akiyama's SU(3) Racah coefficients $U(.$.$) used here are based on Biedenharn and Louck's (Louck 1970) canonical$ resolution for the outer unitary coupling.

There are only two possibilities for the product $[T \times \mathscr{Y}]$. Either

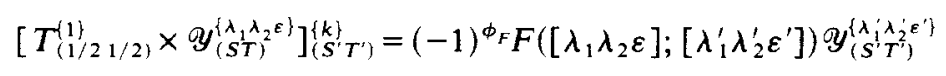

with $\{k\}=\left\{\lambda^{\prime}\right\}$ or

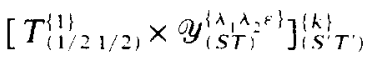

$$
\begin{aligned}
& =(-1)^{\phi_{G}} G\left(\left[\lambda_{1} \lambda_{2} \varepsilon\right] ;\left[\lambda_{1}^{\prime} \lambda_{2}^{\prime} \varepsilon^{\prime}\right] ;\{k\}\right)\left[Z^{\{2\}} \times \mathcal{Y}_{\left(S^{\prime} T^{\prime}\right)}^{\left\{\lambda^{\prime} \varepsilon^{\prime}\right\}}\right]_{\left(S^{\prime} T^{\prime}\right)}^{\{k\}}
\end{aligned}
$$

Note that equations (4.6) and (4.7) would be independent of the $\mathrm{SU}(2) \times \mathrm{SU}(2)$ labels $S, S^{\prime}, T, T^{\prime}$ if the lower coupling were an $\mathrm{O}(4)$ coupling. But since $\mathrm{SU}(2) \times \mathrm{SU}(2)$ is a subgroup of $\mathrm{O}(4)$, these equations do present an $S T$ dependence in the form of the phase factors $(-1)^{\infty}$ included in table 1 .

The functional dependence of $F$ and $G$ on the labels $[\lambda],\left[\lambda^{\prime}\right]$ and $\{k\}$ is quite simple and can be extracted from the very explicit state construction. Furthermore, $G$ can be related to $F$ by application of the symplectic raising operator $B$ (equation (3.17)) on both sides of equation (4.7): by reordering the resulting left-hand side, using the commutator relation

$$
\left[B^{\{00-2\}}, T_{[1](1 / 21 / 2)}^{\{100\}}=2 T_{[1](1 / 21 / 2)}^{\{00-1\}}\right.
$$

where (see Draayer and Akiyama (1973) for conjugation properties of SU(3) tensors)

$$
T_{[1] m_{,} m_{i}}^{\{00-1\} m}=(-1)^{\phi(m)}(-1)^{1-m_{1}-m_{1}}\left(T_{[1]-m_{1}-m_{i}}^{\{100 \mid \tilde{m}}\right)^{i}
$$

and using the symplectic reduced matrix elements (Rowe 1984), one finds

$$
\begin{aligned}
&\left.(-1)^{\phi_{G} G(}\left(\lambda_{1} \lambda_{2} \varepsilon\right] ;\left[\lambda_{1}^{\prime} \lambda_{2}^{\prime} \varepsilon^{\prime}\right] ;\{k\}\right) \\
&=\frac{(-1)^{\left(\lambda_{1}^{\prime}+1-\lambda_{1}\right)}}{\kappa_{\{2\}\{2\}}^{2}\left(\left[\lambda_{1}^{\prime} \lambda_{2}^{\prime} \varepsilon^{\prime}\right] ;\{k\}\right)}\left(\frac{2\left(\lambda_{1}+\lambda_{2}+1-\varepsilon\right)\left(\lambda_{1}-\lambda_{2}+1+\varepsilon\right)}{\left(\lambda_{1}^{\prime}+\lambda_{2}^{\prime}+1-\varepsilon^{\prime}\right)\left(\lambda_{1}^{\prime}-\lambda_{2}^{\prime}+1+\varepsilon^{\prime}\right)}\right)^{1 / 2} \\
& \times(-1)^{\phi_{F}} F\left(\left[\lambda_{1}^{\prime} \lambda_{2}^{\prime} \varepsilon^{\prime}\right] ;\left[\lambda_{1} \lambda_{2} \varepsilon\right]\right) U\left(\left(\mu_{k} \lambda_{k}\right)(10)\left(\lambda_{2}^{\prime}-\varepsilon^{\prime}, \lambda_{1}^{\prime}-\lambda_{2}^{\prime}\right)(10) ;\right. \\
&\left.\times\left(\lambda_{2}-\varepsilon, \lambda_{1}-\lambda_{2}\right)(20)\right)
\end{aligned}
$$


Table 1. Table of $\left.\left[T_{(1 / 21 / 2)}^{\{1\}} \times y_{(S T)}^{\{\lambda\}}\right]_{(S T}^{\{k\}}\right)^{\dagger}$

$$
\begin{aligned}
& {\left[T^{\{1)} \times g^{\left(\lambda_{1} \lambda_{2}\right\}}\right]_{\left(S_{1}^{\prime}=S+1, \lambda_{1}\right\}}^{\left\{\lambda_{2}, T^{\prime}=T+1 / 2\right)}=\left(\frac{\left(\lambda_{1}-\lambda_{2}+1\right)\left(\lambda_{1}+2\right)}{\left(\lambda_{1}-\lambda_{2}+2\right)}\right)^{1 / 2} y_{\left(S T^{\prime}\right)}^{\left\{\lambda_{1}+1, \lambda_{2}\right\}}}
\end{aligned}
$$

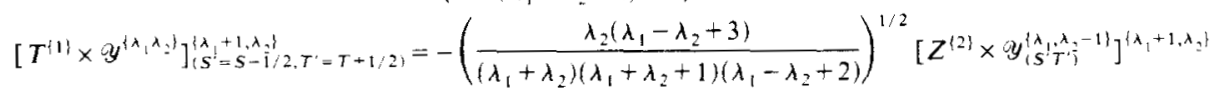

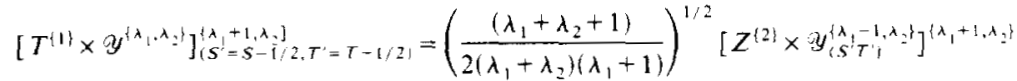

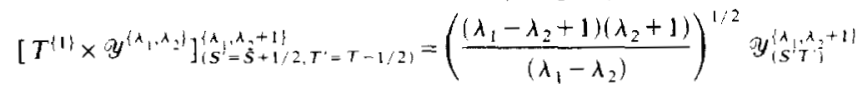

$$
\begin{aligned}
& {\left[T^{\{1\}} \times \mathscr{Y}^{\left\{\lambda_{1}, \lambda_{2}\right\}}\right]_{\left\{S^{\prime}=\bar{S}-1 / 2, T=T+1 / 2\right\}}^{\left\{\lambda_{2}+1\right\}}=-\left(\frac{\left(\lambda_{1}+\lambda_{2}+1\right)}{2 \lambda_{2}\left(\lambda_{1}+\lambda_{2}\right)}\right)^{1 / 2}\left[Z^{\{2\}} \times \mathscr{Y}_{\left\{S^{\prime}\right.}^{\left\{\lambda^{\prime}, \lambda^{\prime},-1\right\}}\right]^{\left\{\lambda_{1}, \lambda_{2}+1\right\}}} \\
& {\left[T^{\{1\}} \times \mathcal{Y}^{\left\{\lambda_{1}, \lambda_{2}\right\}}\right]_{\left\{S^{\prime}=\bar{S}-1 / 2, T^{\prime}=T-1 / 2\right\}}^{\left\{\lambda_{1}+1\right\}}=\left(\frac{\left(\lambda_{1}+1\right)\left(\lambda_{1}-\lambda_{2}-1\right)}{\left(\lambda_{1}+\lambda_{2}+1\right)\left(\lambda_{1}+\lambda_{2}\right)\left(\lambda_{1}-\lambda_{2}\right)}\right)^{1 / 2}\left[Z^{\{2\}} \times Y_{\{}^{\left\{\lambda_{1}-T_{1}, \lambda_{2}\right\}}\right]^{\left\{\lambda_{1}, \lambda_{2}+1\right\}}}
\end{aligned}
$$

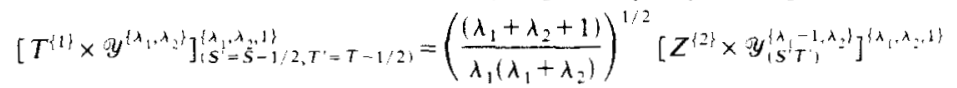

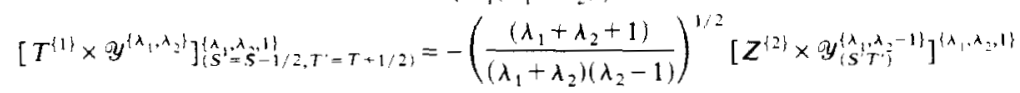

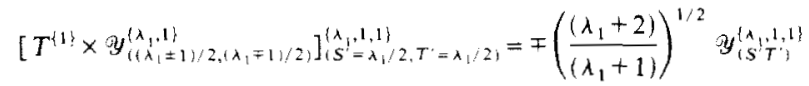

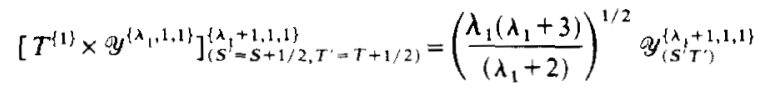

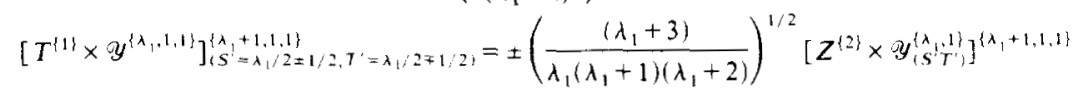

$$
\begin{aligned}
& {\left[T^{(1)} \times \mathcal{O}^{\left\{\lambda_{1}, 1,1\right\}}\right]_{\left(S^{\prime}=\lambda_{1} / 2-1 / 2, T^{\prime}=\lambda_{1} / 2-1 / 2\right)}^{\left\{\lambda_{1}+1,1\right\}}=\frac{1}{\lambda_{1}}\left(\frac{\left(\lambda_{1}-1\right)\left(\lambda_{1}+2\right)}{2\left(\lambda_{1}+1\right)}\right)^{1 / 2}\left[Z^{\{2\}} \times \mathcal{Y}_{\left(S^{\prime} T^{\prime}\right)}^{\left\{\lambda_{1}-1,1,1\right\}}\right]^{\left\{\lambda_{1}+1,1,1\right\}}}
\end{aligned}
$$

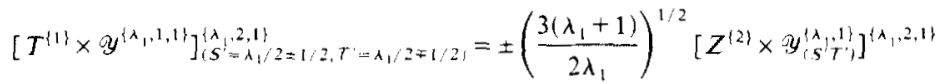

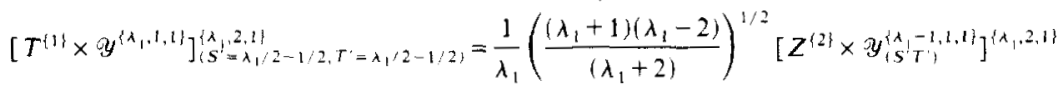

$\dagger$ Note that the quantum numbers $S, T$ will be omitted whenever there is no possible ambiguity in the assignment of their values.

where the $U(3)$ Racah coefficients necessary to evaluate (4.9) are listed in table 1 of Vergados (1968). Altogether, there are 14 different possible cases for equations (4.6) and (4.7) which are listed in table 1.

In case of equation (4.6), we are led directly to the needed reduced matrix element (4.2) and hence the desired SU(4) Wigner coefficient:

$$
\begin{aligned}
\left\langle\begin{array}{c}
\left\{h^{\prime}\right\} j \\
{\left[\lambda^{\prime}\right]\left(S^{\prime} T^{\prime}\right)}
\end{array}\right. & \left.T_{[1\} ; 1 / 21 / 2)}^{\{100\}} \| \begin{array}{c}
\{h\} i \\
{[\lambda](S T)}
\end{array}\right) \\
= & \sum_{\{n\} \rho \rho^{\prime}} \kappa_{\{n\} \rho^{\prime}, j}\left(\left[\lambda^{\prime}\right] ;\left\{h^{\prime}\right\}\right) \kappa_{i,\{n\} \rho}^{-1}([\lambda] ;\{h\}) \\
& \times(-1)^{\varepsilon+\varepsilon^{+}+\phi F} F\left(\left[\lambda_{1} \lambda_{2} \varepsilon\right] ;\left[\lambda_{1}^{\prime} \lambda_{2}^{\prime} \varepsilon^{\prime}\right]\right) \\
& \times U\left(\left(\lambda_{n} \mu_{n}\right)\left(\lambda_{1}-\lambda_{2}, \lambda_{2}-\varepsilon\right)\left(\lambda_{h^{\prime}} \mu_{h^{\prime}}\right)(10) ;\left(\lambda_{h} \mu_{h}\right) \rho_{-}\left(\lambda_{1}^{\prime}-\lambda_{2}^{\prime}, \lambda_{2}^{\prime}-\varepsilon^{\prime}\right) \ldots \rho^{\prime}\right) .
\end{aligned}
$$


For the case of equation (4.7) one more $\mathrm{U}(3)$ recoupling transformation is needed to combine $\left[Z^{\{n\}} \times Z^{\{2\}}\right]$ into $Z^{\left\{n^{\prime}\right\}}$. Together with equation (3.26), this leads to the reduced matrix element

$$
\begin{aligned}
\left\langle\begin{array}{c}
\left\{h^{\prime}\right\} j \\
{\left[\lambda^{\prime}\right]\left(S^{\prime} T^{\prime}\right)}
\end{array}\right. & \left.T_{[1](1 / 21 / 2)}^{\{100\}} \| \begin{array}{c}
\{h\} i \\
{[\lambda](S T)}
\end{array}\right) \\
= & \sum_{\{n\}\left\{n^{\prime}\right\}\{k\} \rho \rho^{\prime} \rho^{\prime \prime}} \kappa_{\left\{n^{\prime}\right\} \rho^{\prime}, j}\left(\left[\lambda^{\prime}\right] ;\left\{h^{\prime}\right\}\right) \kappa_{i,\{n\} \rho}^{-1}([\lambda] ;\{h\}) \\
& \times(-1)^{\varepsilon+\lambda_{i}-\lambda_{k}-\mu_{k}+\phi_{G}} G\left(\left[\lambda_{1} \lambda_{2} \varepsilon\right] ;\left[\lambda_{1}^{\prime} \lambda_{2}^{\prime} \varepsilon^{\prime}\right] ;\{k\}\right)\left(\left\{n^{\prime}\right\}\left\|a^{\dagger}\right\|\{n\}\right) \\
& \times U\left(\left(\lambda_{n} \mu_{n}\right)\left(\lambda_{1}-\lambda_{2}, \lambda_{2}-\varepsilon\right)\left(\lambda_{h^{\prime}} \mu_{h^{\prime}}\right)(10) ;\left(\lambda_{h} \mu_{h}\right) \rho_{-}\left(\lambda_{k} \mu_{k}\right)_{-} \rho^{\prime \prime}\right) \\
& \times U\left(\left(\lambda_{n} \mu_{n}\right)(20)\left(\lambda_{h^{\prime}} \mu_{h^{\prime}}\right)\left(\lambda_{1}^{\prime}-\lambda_{2}^{\prime}, \lambda_{2}^{\prime}-\varepsilon^{\prime}\right) ;\left(\lambda_{n} \mu_{n^{\prime}}\right)_{-} \rho ;\left(\lambda_{k} \mu_{k}\right)_{-} \rho^{\prime \prime}\right) .
\end{aligned}
$$

\subsection{Wigner coefficients for the coupling $\{P+1, P\} \otimes\{1\} \rightarrow\{P+2, P\}$}

Equations (4.2), (4.3), (4.10) and (4.11) make it possible to evaluate the most general SU(4) Wigner coefficient for the coupling $\{h\} \otimes\{1\} \rightarrow\left\{h^{\prime}\right\}$. For the SU(4) representation $\{h\}\left(\right.$ or $\left.\left\{h^{\prime}\right\}\right)=\{P P\},\{P+1, P\},\{P\},\{P, 1\}$ and $\{P, 1,1\}, S$ and $T$ are sufficient labels and the Wigner coefficients for the fundamental tensor between representations of this type have previously been given in general algebraic form (Hecht and Pang 1969). The present method reproduces these results except for the new phase conventions.

Representations of the type $\{P+2, P\},\{P+1, P, 1\},\{P, 2\}$ have at most twofold multiplicities in $S, T$. These cases exhaust most of the possibilities which may be needed for the nuclear $\mathrm{p}$ and sd shells and will illustrate how the new method can resolve the multiplicities.

4.2.1. Coefficients for the coupling $\{P+1, P\} \otimes\{1\} \rightarrow\{P+2, P\}\left(P-\lambda_{1}\right.$ odd $)$. If $P-\lambda_{1}$ is odd, the $O(4)$ representation $\left[\lambda_{1} \lambda_{2}\right]$ will appear only once in the decomposition

$$
\mathrm{SU}(4) \downarrow \mathrm{O}(4):\{P+2, P\} \downarrow\left[\lambda_{1} \lambda_{2}\right] \text {. }
$$

Equations (4.2), (4.3), (4.10) and (4.11) then lead directly to the results of table $3(a)$. Note that the needed $\kappa$ matrices are then one dimensional and can easily be obtained recursively (Rowe 1984). They are listed in table 2. Note also that table 3 gives the Wigner coefficients in terms of the $O(4)$ labels $\lambda_{1}$ and $\lambda_{2}$. To obtain the coefficients corresponding to the specific $S U(2) \times S U(2)$ subgroups $S, T=\frac{1}{2}\left(\lambda_{1} \pm \lambda_{2}, \frac{1}{2} \lambda_{1} \mp \lambda_{2}\right)$, one only has to make the substitution $\lambda_{1}=S+T$ and $\lambda_{2}= \pm(S-T)$ in these expressions. For example, one obtains (for $P-\lambda_{1}$ odd)

$$
\begin{gathered}
\left\langle\begin{array}{cc}
\{P+1, P\} & \{1\} \\
{\left[\lambda_{1}, \lambda_{2}+1\right]} & \left(S^{\prime}=S+\frac{1}{2}, T^{\prime}=T-\frac{1}{2}\right)^{\prime}[1]
\end{array} \mid \begin{array}{c}
\{P+2, P\} \\
{\left[\lambda_{1} \lambda_{2}\right]\left(S=\frac{1}{2}\left(\lambda_{1}+\lambda_{2}\right), T=\frac{1}{2}\left(\lambda_{1}-\lambda_{2}\right)\right)}
\end{array}\right\rangle \\
=\left(\frac{(P-S+T+2) S(T+1)}{(P+3)(2 S+1)(2 T+1)}\right)^{1 / 2} \\
\left\langle\begin{array}{cc}
\{P+1, P\} & \{1\} \\
{\left[\lambda_{1}, \lambda_{2}-1\right]\left(S^{\prime}=S-\frac{1}{2}, T^{\prime}=T+\frac{1}{2}\right)^{\prime}[1]}
\end{array} \|_{\left[\lambda_{1} \lambda_{2}\right]\left(S=\frac{1}{2}\left(\lambda_{1}+\lambda_{2}\right), T=\frac{1}{2}\left(\lambda_{1}-\lambda_{2}\right)\right)}\right\rangle \\
=-\left(\frac{(P+S-T+2) T(S+1)}{(P+3)(2 S+1)(2 T+1)}\right)^{1 / 2} .
\end{gathered}
$$


Table 2. Table of $\kappa^{2}([\lambda] ;\{h\}), \lambda_{1}-\lambda_{2}$ even integer.

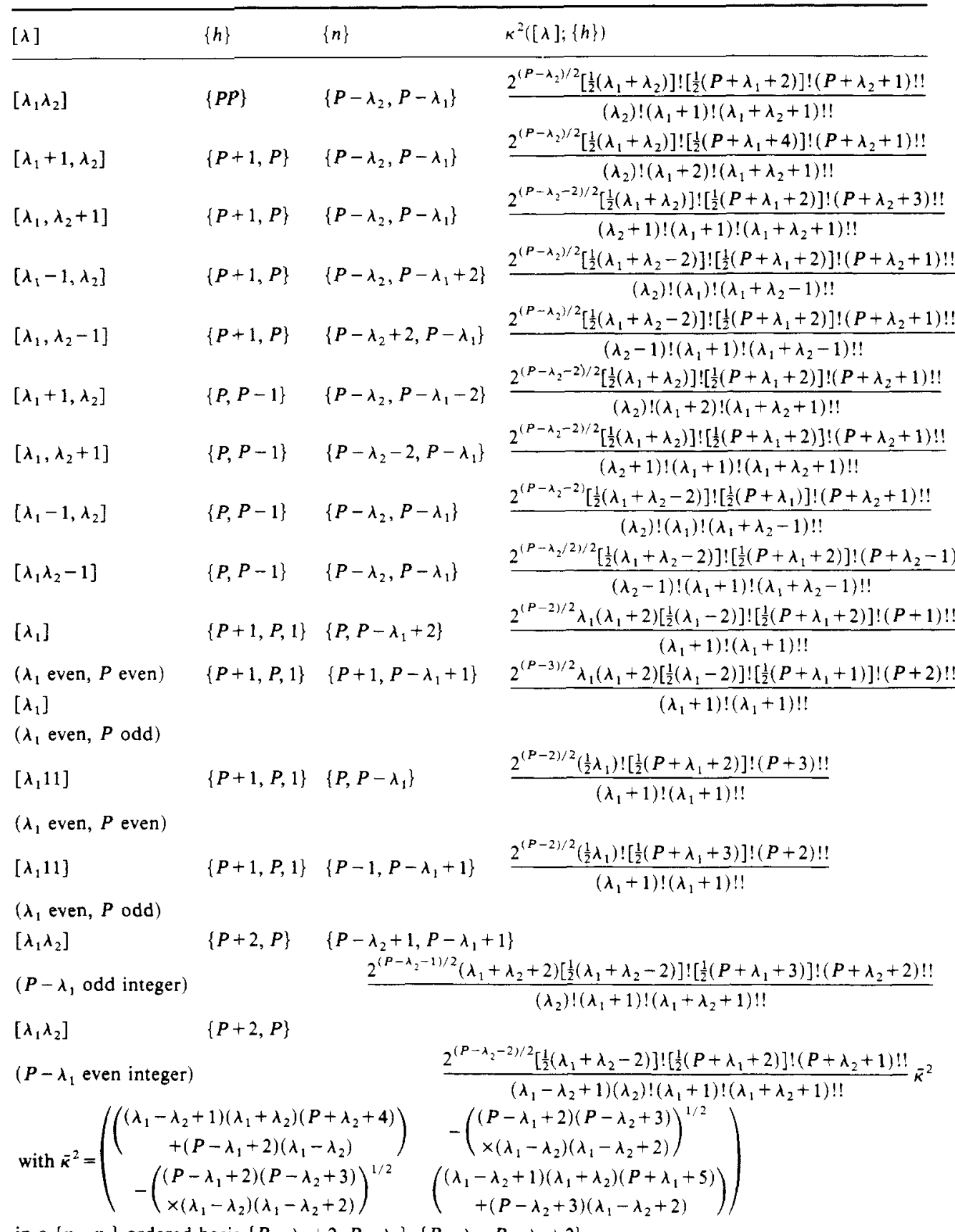

in a $\left\{n_{1}, n_{2}\right\}$ ordered basis $\left\{P-\lambda_{2}+2, P-\lambda_{1}\right\},\left\{P-\lambda_{2}, P-\lambda_{1}+2\right\}$ 


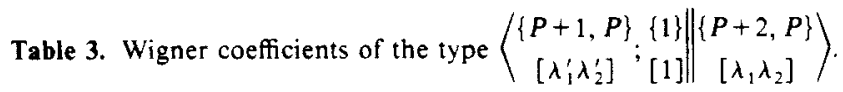

(a) $\left(P-\lambda_{1}\right.$ odd $)$

\begin{tabular}{|c|c|}
\hline$\left[\lambda_{1}^{\prime} \lambda_{2}^{\prime}\right]$ & $\left\langle\begin{array}{c}\{P+1, P\} \\
{\left[\lambda_{1}^{\prime} \lambda_{2}^{\prime}\right]}\end{array}: \begin{array}{c}\{1\} \\
{[1]}\end{array} \|\left\{\begin{array}{c}\{P+2, P\} \\
{\left[\lambda_{1} \lambda_{2}\right]}\end{array}\right\rangle\right.$ \\
\hline$\left[\lambda_{1}+1, \lambda_{2}\right]$ & $\left(\frac{\left(P-\lambda_{1}+1\right)\left(\lambda_{1}+\lambda_{2}\right)\left(\lambda_{1}-\lambda_{2}\right)}{4(P+3)\left(\lambda_{1}+\lambda_{2}+1\right)\left(\lambda_{1}-\lambda_{2}+1\right)}\right)^{1 / 2}$ \\
\hline$\left[\lambda_{1}, \lambda_{2}+1\right]$ & $\left(\frac{\left(P-\lambda_{2}+2\right)\left(\lambda_{1}+\lambda_{2}\right)\left(\lambda_{1}-\lambda_{2}+2\right)}{4(P+3)\left(\lambda_{1}+\lambda_{2}+1\right)\left(\lambda_{1}-\lambda_{2}+1\right)}\right)^{1 / 2}$ \\
\hline$\left[\lambda_{1}-1, \lambda_{2}\right]$ & $\left(\frac{\left(P+\lambda_{1}+3\right)\left(\lambda_{1}+\lambda_{2}+2\right)\left(\lambda_{1}-\lambda_{2}+2\right)}{4(P+3)\left(\lambda_{1}+\lambda_{2}+1\right)\left(\lambda_{1}-\lambda_{2}+1\right)}\right)^{1 / 2}$ \\
\hline$\left[\lambda_{1}, \lambda_{2}-1\right]$ & $-\left(\frac{\left(P+\lambda_{2}+2\right)\left(\lambda_{1}+\lambda_{2}+2\right)\left(\lambda_{1}-\lambda_{2}\right)}{4(P+3)\left(\lambda_{1}+\lambda_{2}+1\right)\left(\lambda_{1}-\lambda_{2}+1\right)}\right)^{1 / 2}$ \\
\hline
\end{tabular}

(b) $\left(P-\lambda_{1}\right.$ even $)$

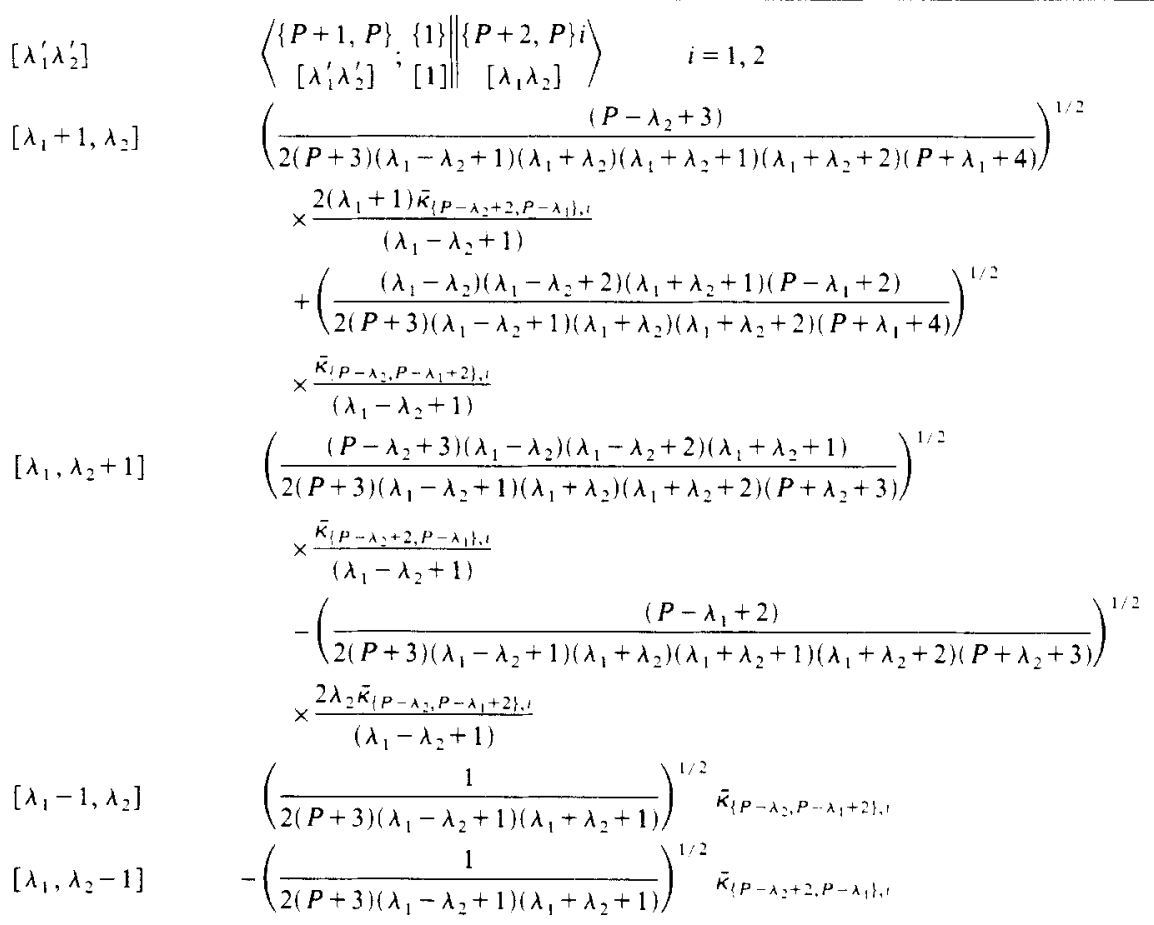

4.2.2. Coefficients for the coupling $\{P+1, P\} \otimes\{1\} \rightarrow\{P+2, P\}\left(P-\lambda_{1}\right.$ even $)$. If $P-\lambda_{1}$ is even, the $O(4)$ representation $\left[\lambda_{1} \lambda_{2}\right]$ will appear twice in the decomposition

$$
\mathrm{SU}(4) \downarrow \mathrm{O}(4):\{P+2, P\} \downarrow\left[\lambda_{1} \lambda_{2}\right]
$$

except if $\lambda_{1}=\lambda_{2}$ or $\lambda_{1}=P+2$ for which it appears only once. We find for the general twofold case the results of table $3(b)$. Note that the $2 \times 2$ matrix $\bar{\kappa}^{2}$ of table $3(b)$ is defined in table 2 and is diagonalised by the Hermitian operator $U$ with

$$
\Lambda=U \bar{\kappa}^{2} U^{\dagger} \quad \bar{\kappa}_{\{n\}, i}=U_{\{n\}, i} \Lambda_{i}^{1 / 2}
$$


with

$$
U=U^{*}=\left(\begin{array}{rr}
\cos \theta & \sin \theta \\
\sin \theta & -\cos \theta
\end{array}\right) .
$$

For $\lambda_{1}=P+2$, only the zero-coupled pair polynomial of rank $\left\{n_{1}, n_{2}\right\}=\left\{P-\lambda_{2}, 0\right\}$ occurs and the overlap matrix becomes one dimensional:

$$
\bar{\kappa}^{2} \rightarrow 2(P+3)\left(P+\lambda_{2}+3\right)\left(P-\lambda_{2}+3\right)
$$

(see table 2). The only non-vanishing Wigner coefficient is then given by

$$
\left\langle\begin{array}{c}
\{P+1, P\} \\
{\left[\lambda_{1}-1, \lambda_{2}\right]}
\end{array} ; \begin{array}{c}
\{1\} \\
{[1]}
\end{array} \|\left(\begin{array}{c}
\{P+2, P\} \\
{\left[\lambda_{1} \lambda_{2}\right]}
\end{array}\right\rangle=+1 .\right.
$$

For $\lambda_{1}=\lambda_{2}$, only $\left\{n_{1}, n_{2}\right\}=\left\{P-\lambda_{1}+2, P-\lambda_{1}\right\}$ occurs and

$$
\vec{\kappa}^{2} \rightarrow 2 \lambda_{1}\left(P+\lambda_{1}+4\right)
$$

(see table 2). The only non-vanishing Wigner coefficients are then given by

$$
\begin{aligned}
& \left\langle\begin{array}{c}
\{P+1, P\},\{1\} \\
{\left[\lambda_{1}+1, \lambda_{1}\right] ;[1]}
\end{array} \|\left\{\begin{array}{c}
\{P+2, P\} \\
{\left[\lambda_{1} \lambda_{1}\right]}
\end{array}\right\rangle=\left(\frac{\left(\lambda_{1}+1\right)\left(P-\lambda_{1}+3\right)}{(P+3)\left(2 \lambda_{1}+1\right)}\right)^{1 / 2}\right. \\
& \left\langle\begin{array}{c}
\{P+1, P\},\{1\} \\
{\left[\lambda_{1}, \lambda_{1}-1\right],[1]}
\end{array} \|\left\{\begin{array}{c}
\{P+2, P\} \\
{\left[\lambda_{1} \lambda_{1}\right]}
\end{array}\right\rangle=-\left(\frac{\lambda_{1}\left(P+\lambda_{1}+4\right)}{(P+3)\left(2 \lambda_{1}+1\right)}\right)^{1 / 2}\right. \text {. }
\end{aligned}
$$

\subsection{Wigner coefficients for the coupling $\{P+1, P\} \times\{1\} \rightarrow\{P+1, P, 1\}$}

As a final example, we will give numerical values for Wigner coefficients with a final SU(4) of the type $\{P+1, P, 1\}$ with $S=T$ where a twofold multiplicity is resolved by the $O(4)$ labels: one state being of $O(4)$ rank $\left[\lambda_{1}\right]$, the other being of $O(4)$ rank $\left[\lambda_{1} 11\right]$ so that the $\kappa^{2}$ matrices are one dimensional. More specifically, we choose $P=2$, $(S T)=\left(\frac{1}{2} \frac{1}{2}\right)$ because it is the only case where a twofold multiplicity can occur in the nuclear $\mathrm{p}$ shell.

Since we know the general Wigner coefficient for the coupling:

$$
\{P+1, P\} \otimes\{1\} \rightarrow\{P+2, P\}
$$

( $\$ 4.2$ ), we can write down the full $4 \times 4$ matrix for

$$
\left\langle\begin{array}{cc}
\{3,2\} & \{1\} \\
\left(S^{\prime} T^{\prime}\right) & \left(\frac{1}{2} \frac{1}{2}\right)
\end{array} \| \begin{array}{c}
\{h\} i \\
(S T)=(11)
\end{array}\right\rangle
$$

as follows:

$$
\begin{array}{ccccc}
\frac{\{h\}[\lambda] i}{\left(S^{\prime} T^{\prime}\right)}\{321\}[2] & \{321\}[211] & \{42\}[2] i=1 & \{42\}[2] i=2 \\
\left(\frac{1}{2} \frac{1}{2}\right) & \frac{2 \sqrt{ } 2}{3 \sqrt{ } 5} & 0 & \frac{\sin \theta \sqrt{ } \Lambda_{-}}{3 \sqrt{ } 10} & -\frac{\cos \theta \sqrt{ } \Lambda_{+}}{3 \sqrt{ } 10}
\end{array}
$$




$\begin{array}{ccccc}\left(\frac{3}{2} \frac{1}{2}\right) & \frac{1}{3 \sqrt{ } 2} & \frac{1}{\sqrt{ } 2} & \frac{\cos \theta \sqrt{ } \Lambda_{-}}{3 \sqrt{ } 10} & \frac{\sin \theta \sqrt{ } \Lambda_{+}}{3 \sqrt{ } 10} \\ \left(\frac{1}{2} \frac{3}{2}\right) & \frac{1}{3 \sqrt{ } 2} & -\frac{1}{\sqrt{ } 2} & \frac{\cos \theta \sqrt{ } \Lambda_{-}}{3 \sqrt{ } 10} & \frac{\sin \theta \sqrt{ } \Lambda_{+}}{3 \sqrt{ } 10} \\ \left(\frac{3}{2} \frac{3}{2}\right) & -\frac{4 \sqrt{ } 2}{3 \sqrt{ } 5} & 0 & \left(\frac{\cos \theta}{12 \sqrt{ } 2}+\frac{\sin \theta}{6 \sqrt{ } 10}\right) \sqrt{ } \Lambda_{-} & \left(\frac{\sin \theta}{12 \sqrt{ } 2}-\frac{\cos \theta}{6 \sqrt{ } 10}\right) \sqrt{ } \Lambda_{+}\end{array}$

with

$$
\Lambda_{ \pm}=(57 \pm \sqrt{ } 369)
$$

and

$$
\frac{\cos \theta}{\sin \theta}=\left[\left(\frac{1}{2} \pm \frac{17}{2 \sqrt{369}}\right)\right]^{1 / 2}
$$

(Note that while Jahn and van Wieringen (1951) had to make an arbitrary choice in their definition of the two $\{321\}(S T)=(11)$ states, the present resolution of the twofold multiplicity through the use of the [2] and [211] $\mathrm{O}(4)$ labels gives an unambiguous definition of the states and therefore of the corresponding Wigner coefficients. Note that this choice automatically results in the vanishing of two coefficients.)

\section{Matrix elements for the $S U(4)$ generators in an $S U(4) \supset O(4)$ basis}

Matrix elements for more general $S U(4) \supset O(4)$ tensors are easily obtained using a buildup process where the basic building block is the fundamental tensor $T_{[1\}}^{\{1\}}$. In this final section, we give a simple algorithm for the computation of the generators of the Lie algebra $\mathrm{SU}(4)$ in an $\mathrm{SU}(4) \supset \mathrm{O}(4)$ basis using such a technique.

The generators $E_{i s i}$ with $(s t)=(11),(10)$ and $(01)$ can be expressed as the tensorial coupled product of $T_{[1]}^{\{100\}}(z)(\sim z)$ with its Hermitian conjugate $T_{[1]}^{\{00-1\}}(z)(\sim \partial / \partial z)$ :

$$
\begin{aligned}
& E_{\text {sm, }, m_{1}}=\sum_{\alpha} \sum_{m_{1}, m_{r_{2}, m_{1}, m_{i_{2}}}}(-1)^{1-m_{r_{2}}-m_{l_{2}}} \\
& \times\left\langle\frac{1}{2} m_{s_{1}} ; \frac{1}{2} m_{s_{2}} \mid s m_{1}\right\rangle\left\langle\frac{1}{2} m_{t_{1}} ; \frac{1}{2} m_{t_{2}} \mid t m_{t}\right\rangle z_{\alpha m_{1}, m_{1}} \partial / \partial z_{\alpha-m_{12}-m_{t_{2}}} .
\end{aligned}
$$

(Note that $E$ is a $\mathrm{U}(3)$ scalar operator while it transforms as a $\{100-1\} \sim\{211\}$ tensor under U(4).)

Since matrix elements of $E_{(10)} \sim S$ and of $E_{(01)} \sim T$ are easily obtained from angular momentum calculus (Hecht and Pang 1969), we only need to specifically work out those of $E_{(11)}$. Using hermiticity, we find

$$
\begin{aligned}
& \left\langle\begin{array}{c}
\left\{h^{\prime}\right\} j \\
{\left[\lambda^{\prime}\right]\left(S^{\prime} T^{\prime}\right)}
\end{array}\left\|T_{[1](1 / 21 / 2)}^{\{100\}}\right\| \begin{array}{c}
\{h\} i \\
{[\lambda](S T)}
\end{array}\right\rangle \\
& =(-1)^{\lambda_{1}-\lambda_{1}+h_{1}-h_{3}-h_{1}^{\prime}+h_{3}^{\prime}}\left(\frac{\operatorname{dim}\left(\lambda_{h} \mu_{h}\right)(2 S+1)(2 T+1)}{\operatorname{dim}\left(\lambda_{h} \cdot \mu_{h^{\prime}}\right)\left(2 S^{\prime}+1\right)\left(2 T^{\prime}+1\right)}\right)^{1 / 2} \\
& \times\left\langle\begin{array}{c}
\{h\}_{i} \\
{[\lambda](S T)}
\end{array}\left\|T_{[1](1 / 21 / 2)}^{\{00-1\}}\right\| \begin{array}{c}
\left\{h^{\prime}\right\} j \\
{\left[\lambda^{\prime}\right]\left(S^{\prime} T^{\prime}\right)}
\end{array}\right)
\end{aligned}
$$


from which we deduce the following $S U(4) \supset O(4)$ reduced matrix elements for the generators:

$$
\begin{aligned}
& \left\langle\begin{array}{c}
\{h\} j \\
{\left[\lambda^{\prime}\right]\left(S^{\prime} T^{\prime}\right)}
\end{array}\left\|E_{(11)}\right\| \begin{array}{c}
\{h\} i \\
([\lambda](S T)
\end{array}\right) \\
& =\sum_{\left.\left\{h^{\prime \prime}\right\}\right\}^{\prime \prime}\left[\lambda^{\prime \prime}\right] S^{\prime \prime} T^{\prime \prime}}(-1)^{S+T+1-S^{\prime \prime}-T^{\prime \prime}}\left(\frac{(2 S+1)(2 T+1)}{\left(2 S^{\prime}+1\right)\left(2 T^{\prime}+1\right)}\right)^{1 / 2} \\
& \times U\left(S_{2}^{1} S_{2}^{\prime} ; S^{\prime \prime} 1\right) U\left(T_{2}^{1} T_{2}^{\prime \prime} ; T^{\prime \prime} 1\right)\left\langle\begin{array}{c}
\{h\} j \\
{\left[\lambda^{\prime}\right]\left(S^{\prime} T^{\prime}\right)}
\end{array}\left\|T_{[1] 1 / 21 / 2)}^{\{100\}}\right\| \begin{array}{c}
\left\{h^{\prime \prime}\right\} l^{\prime \prime} \\
{\left[\lambda^{\prime \prime}\right]\left(S^{\prime \prime} T^{\prime \prime}\right)}
\end{array}\right\rangle \\
& \times\left\langle\begin{array}{c|c}
\{h\} i \\
{[\lambda](S T)}
\end{array}\left\|T_{\{1](1 / 21 / 2)}^{\{100\}}\right\| \begin{array}{c}
\left\{h^{\prime \prime}\right\} l^{\prime \prime} \\
{\left[\lambda^{\prime \prime}\right]\left(S^{\prime \prime} T^{\prime \prime}\right)}
\end{array}\right\rangle
\end{aligned}
$$

where $S, T=\frac{1}{2}\left(\lambda_{1} \pm \lambda_{2}\right), \frac{1}{2}\left(\lambda_{1} \mp \lambda_{2}\right)$ for $[\lambda]=\left[\lambda_{1} \lambda_{2}\right]$, etc.

\section{References}

Biedenharn L C, Giovannini A and Louck J D 1967 J. Math. Phys. 8691

Brunet M and Resnikoff M 1970 J. Math. Phys. 11 1471, 1474

Castaños O, Chacón E and Moshinsky M 1984 J. Math. Phys. 251211

Castaños O, Chacón E, Moshinsky M and Quesne C 1985a J. Math. Phys. 262107

Castaños O, Kramer P and Moshinsky M 1985b J. Phys. A: Math. Gen. 18 L493

Deenen J and Quesne C 1983 J. Phys. A: Math. Gen. 16205

Draayer J P 1970 J. Math. Phys. 113225

Draayer J P and Akiyama Y 1973 J. Math. Phys. 141904

Hecht K T 1965 Nucl. Phys. 621

Hecht K T and Pang S C 1969 J. Math. Phys. 101571

Jahn H A and van Wieringen H 1951 Proc. R. Soc. A 209502

Le Blanc R and Rowe D J 1985a J. Phys. A: Math. Gen. 181891

_ 1985 b J. Phys. A: Math. Gen. 181905 1986 J. Phys. A: Math. Gen. 192913

Littlewood D E 1950 The Theory of Group Characters (Oxford: Clarendon)

Lohe M A and Hurst C A 1971 J. Math. Phys. 121882

Louck J D 1970 Am. J. Phys. 383

Louck J D and Galbraith H W 1972 Rev. Mod. Phys. 44540

Moshinsky M 1962 Rev. Mod. Phys. 34813

Moshinsky M and Devi S 1969 J. Math. Phys. 10455

Moshinsky M and Nagel J G 1963 Phys. Lett. 5173

Moshinsky M and Quesne C 1970 J. Math. Phys. 111631

- 1971 J. Math. Phys. 121772

Quesne C 1976 J. Math. Phys. 171452

-_ 1977 J. Math. Phys. 181210

1981 J. Math. Phys. 221482

- 1984a J. Phys. A: Math. Gen. 17777

_ 1984b J. Phys, A: Math. Gen. 17791

Rosensteel G and Rowe D J 1980 Ann. Phys., NY 126343 1983 J. Math. Phys. 242461

Rowe D J 1984 J. Math. Phys. 252662

Rowe D J, Rosensteel G and Carr R 1984 J. Phys. A: Math. Gen. 17 L399

Rowe D J, Wybourne B G and Butler P H 1985 J. Phys. A: Math. Gen. 18939

Vergados J D 1968 Nucl. Phys. A 111681

Wigner E P 1937 Phys. Rev. 51106 